\title{
Synthetic PEG Hydrogels as Extracellular Matrix Mimics for Tissue Engineering Applications
}

\author{
Georgia Papavasiliou*, Sonja Sokic and Michael Turturro \\ Illinois Institute of Technology, Department of Biomedical Engineering, \\ USA
}

\section{Introduction}

In recent years the field of tissue engineering, or regenerative medicine, has developed from the need to replace damaged and/or diseased tissues and organs by combining biomaterial scaffolds, biological signaling molecules, and cells. The regeneration of tissues may be achieved using either one of two principle approaches: 1) the in vitro construction or 2) the in vivo induction of tissue. In the first approach, biomaterial scaffolds are combined with biofunctional signaling molecules and cells and a fully functional tissue is grown in vitro which can then be implanted into the host. In the second approach, scaffolds are tailored with the desired biochemical composition as well as physical and mechanical properties of the target tissue, implanted into the host, and the body is used as a bioreactor to regenerate the tissue of interest. Therefore, biomaterial scaffolds play a central role in regenerative medicine as physical and biochemical milieus that dictate cell behavior, function, and tissue regeneration (Lutolf \& Hubbell, 2005). While both natural and synthetic biomaterials have been extensively explored as scaffolds for tissue regeneration, polymeric materials from synthetic sources are advantageous due to their tunable mechanical properties and ability to systematically and selectively incorporate biological signals of the natural extracellular matrix (ECM) enabling controlled study of cell-substrate interactions. Over the last several decades synthetic crosslinked hydrogels of poly(ethylene) glycol have been extensively investigated for numerous biomedical applications including drug delivery, immunoisolation, and as matrices for engineering tissues. PEG hydrogels are biocompatible, hydrophilic polymers composed of 3D interstitial crosslinks that swell extensively in aqueous environments with water content similar to soft tissues. These biomaterials are inherently resistant to non-specific cell adhesion and protein adsorption, thus providing a blank slate upon which ECM-derived signals can be systematically introduced as well as spatially and temporally manipulated to control cell behavior and tissue regeneration. The continued enhancement of PEG-based biomaterial strategies towards the rational design of scaffolds is highly dependent on their ability to independently control the incorporation of multiple biofunctional signaling molecules from alterations in hydrogel degradation kinetics and mechanical properties, to temporally and spatially tune the presentation of mechanical and biofunctional signals, and to promote rapid and guided neovascularization (new blood vessel formation) prior to complete material degradation. The combination of the above-

${ }^{*}$ Corresponding Author 
mentioned strategies may ultimately result in PEG-based scaffolds that contain the necessary cues that recapitulate the dynamic environment of the ECM ultimately leading to the regeneration of tissues. Here we address common approaches and polymerization techniques used to fabricate PEG scaffolds with 3D spatial and/or temporal variations in physical, mechanical, and biofunctional cues of the native ECM and highlight the important signals embedded in these scaffolds that support cell behavior and tissue regeneration.

\section{The molecular constituents and role of the extracellular matrix}

Since cells receive a myriad of signals from and interact highly with their immediate extracellular microenvironment, the extracellular matrix (ECM), the effective design and improvement of biomaterial scaffold strategies is highly dependent on incorporated knowledge of ECM structure and function. Once believed to function as a passive scaffold for the maintenance of tissue and organ structure, it is now well recognized that the ECM is a dynamic construct that upon its interaction with cells undergoes constant remodeling (i.e. assembly and degradation of its constituents) particularly during the normal physiological process of development, differentiation, and wound healing (Daley et al., 2008). The ECM is composed of macromolecular constituents that are primarily produced locally by the cells that surround it. In most connective tissues, ECM macromolecules are secreted primarily by fibroblast cells, while in connective tissues of bone and cartilage the macromolecular constituents are secreted by osteoblasts and chondrocytes, respectively (Alberts et al, 2008). The macromolecular constituents of the ECM are composed of a three-dimensional array of protein fibers and filaments embedded in a hydrated gelatinous network of polysaccharide chains of glycosaminoglycans (GAGs) and proteoglycans (Fig. 1).

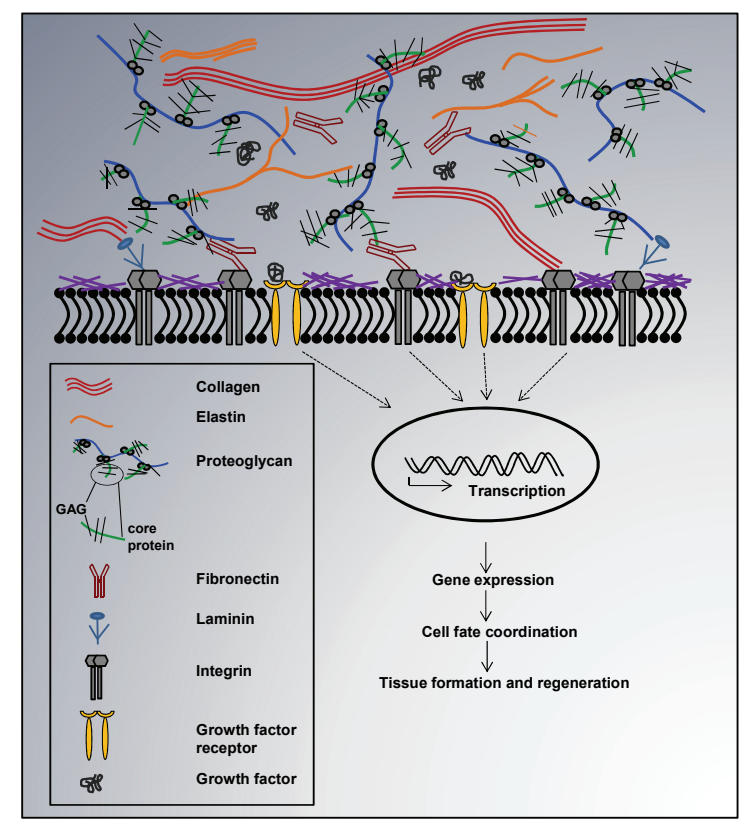

Fig. 1. Schematic illustration of key ECM components and their interactions with cells. 
The ECM also contains growth factors and other bioactive molecules, as well as binding sites for cell-surface molecules exposed upon proteolysis (Somerville et al., 2003). Macromolecular GAGs are hydrophilic, unbranched, negatively charged polysaccharide chains that form stiff and highly extended conformations. Their negative charges attract counterions inducing an osmotic effect that enables the matrix to occupy large volumes of water. The four main groups of GAGs are hyaluronic acid, chondroitin sulfate, keratin sulfate, and heparin sulfate. Most GAGs are sulfated, covalently linked to protein via linker proteins and are synthesized intracellularly and released via exocytosis. The exception among the GAGs is hyaluronic acid which is released directly from the cell surface by an enzyme complex embedded in the plasma membrane, is unsulfated, not covalently linked to proteins, and free of sugar groups (Alberts et al., 2008; Saltzman, 2004). Hyaluronic acid is found in abundant quantities during embryogenesis and wound healing where its presence modulates cell migration in the extracellular space by controlling the level of hydration in tissues. Proteoglycans have extremely high sugar content $(\sim 95 \%)$ and are composed of a core protein to which GAGs are attached via linker proteins (Fig. 1). The GAG chains of proteoglycans enable them to form gels of varying porosity and charge density allowing them to serve as filters that regulate the molecular diffusion of molecules and cells. This structural and chemical organization allows proteoglycans to function as mediators of cell adhesion, regulators of growth factor secretion, and activators of secretion of proteolytic enzymes and protease inhibitors that are involved in both the assembly and degradation of ECM components.

The proteins of the ECM can be classified into structural (i.e., collagen and elastin) and adhesive (i.e., fibronectin and laminin) types. The most abundant structural protein in the ECM is collagen, which is secreted in large quantities primarily by cells of connective tissues. Various chemically distinct forms of collagen exist, each with a basic macromolecular unit comprised of an $\alpha$ triple helical structure composed of three polypeptide chains of $\sim 1000$ amino acids each that are specific to the type of collagen (Patino et al., 2002). Collagen assembles into different supramolecular structures allowing it to have extraordinary functional diversity. The most common forms of collagen found in the ECM are types I, II, III, and IV. Collagens I, II, and III are of the fibrillar type where upon secretion into the ECM organize into higher order polymer structures composed of long (several microns in length) and thin collagen fibrils (10-300 nm in diameter). Collagen assembly into fibrils and fibers is stabilized by covalent crosslinks formed by lysine residues between constituent collagen molecules (Saltzman, 2004). These crosslinks provide collagen fibrils with the tensile strength required to resist tensile forces. Fibrillar collagens interact with cells through integrin receptors on the cell membrane (Fig. 1) to induce cell differentiation and migration during embryonic development. Collagen type IV is a network forming collagen resulting in a mesh-like lattice forming a major portion of the basal lamina which separates epithelial sheets from other tissues and binds to cells via indirect binding to laminin adhesive proteins (Fig. 1). In healthy tissues, collagen is continuously degraded and replaced with turnover times ranging from months to years depending on the tissue type (Alberts et al., 2008).

Elasticity of the ECM is provided from elastic fibers that are primarily composed of elastin molecules that form crosslinked networks of fibers and sheets that enable them to stretch and relax upon deformation. Similar to collagen IV, these crosslinked structures are formed via covalent bond interactions of lysine residues between individual elastin molecules. This 
anisotropic structure of protein fibers and filaments mechanically and biochemically influences cell behavior. Cells within this microenvironment sense the mechanical properties of the ECM and convert mechanical signals into biochemical signals due to the direct interaction of the ECM with the cytoskeleton via cell-surface receptors (Lutolf \& Hubbell, 2005).

Proteins of the adhesive type possess multiple domains that contain specific binding sites for ECM macromolecules as well as for integrins on the surface of cells. Integrins are a class of cell-surface receptors consisting of two non-covalently associated transmembrane $\alpha$ and $\beta$ heterodimers that contain an extracellular ligand binding domain, a transmembrane domain, and a cytoplasmic domain (Steffensen et al., 2011). Integrin-ECM ligand binding interactions provide communication between the intracellular and extracellular environments which in turn affect cellular fate processes such as proliferation, migration, and differentiation (Fig. 1). Cells adhere by integrin-mediated interactions with ECM adhesion proteins such as fibronectin and laminin (Patterson et al., 2010). During this cellmatrix binding event, the extracellular domain of the integrin receptor binds to adhesive protein oligopeptide regions and the cytoplasmic portion binds to focal adhesions linking the ECM to the cytoskeleton resulting in signal transduction. The adhesive protein fibronectin is a large dimeric glycoprotein composed of two subunits each containing approximately 2500 amino acids linked together by disulfide bonds. Integrin receptors on cell surfaces bind to a fibronectin domain containing the well-known tripeptide adhesion sequence arginine-glycine-aspartic acid (RGD) and the neighboring "synergy site" while other distinct protein domains bind to collagen and heparin (Daley et al., 2008). While most cell types bind to the RGD sequence, an additional region (IIICS) within fibronectin has been identified to contain the peptide sequences REDV and LDV that permit the adhesion of specific cell types such as neural cells and lymphocytes. Laminin, a large cross-shaped adhesion protein composed of $\alpha(400 \mathrm{kDa}), \beta(215 \mathrm{kDa})$, and $\gamma(205 \mathrm{kDa})$ polypeptide subunits linked by disulfide bonds, is primarily associated with basement membranes. Different types of isoforms of laminin exist which are created by the different types of $\alpha, \beta$, and $\gamma$ polypeptide chain interactions. Multiple functional regions have been identified in laminin such as those containing the RGD and YIGSR sequences resulting in cell adhesion, the IKVAV peptide motif involved in neurite growth, and a heparin and collagen IV binding region (Saltzman, 2004).

Within a single tissue the ECM is continuously being remodeled by cells via the degradation of ECM components resulting from the secretion of proteolytic enzymes followed by the reassembly of newly synthesized protein components secreted by cells. Cells adhered to the ECM have to change from an adhesive phenotype to a migratory phenotype before they can migrate within its three-dimensional structure (Vu \& Werb, 2000). During the process of cell migration, cells secrete proteolytic enzymes (proteases) that cleave a variety of ECM substrates to break down physical barriers that inhibit cell locomotion. While there are several families of proteases secreted by cells that are involved in ECM degradation, proteases of the matrix metalloproteinase (MMP) family have been shown to be central to tissue homeostasis and ECM remodeling (Steffensen et al., 2011). MMPs are a family of structurally related endopeptidases comprising 23 enzymes in humans all acting to degrade parts of the ECM. Most MMPs are secreted in latent form as pro-enzymes. The latency of the zymogen is dependent on a "cysteine switch" formed by the interaction of a conserved cysteine on the pro-domain with a catalytic zinc atom within the active site of the enzyme 
blocking access to the catalytic site. Enzyme activation results from disruption of the cysteine-zinc pairing due to displacement of the pro-domain by conformational change or proteolysis induced by the protease plasmin or by other MMPs (Singer \& Clark, 1999). There are four main classes of MMPs: collagenases (MMPs -1, 8, and 13) that degrade fibrillar type collagens I, II and III in their triple helical domains to form gelatin, gelatinases (MMPs- 2 and 9) that degrade gelatin as well as basement membrane proteins and elastin, stromelysins (MMP-3, 10, and 11) which have broad substrate specificity but possess the ability to activate other MMPs, and membrane-type MMPs (MT-MMPs) that are bound to the cell membrane and similar to stromelysins, can activate other MMPs in addition to their ability to degrade ECM components (Somerville et al., 2003).

The ECM also modulates tissue dynamics through its ability to bind, store, and release growth factors (Lutolf et al., 2003; Somerville et al., 2003). Growth factors may be released by cells for immediate signaling or embedded within ECM components and growth factor binding proteins and released upon ECM proteolytic degradation (Chen \& Mooney, 2003). Controlled growth factor release within the ECM is balanced by extracellular degradation. During processes such as wound healing, angiogenesis, and tissue repair, ECM-bound growth factors are released upon MMP proteolytic activity. MMPs can also cleave growth factor receptors on cell surfaces to release growth factor receptor ectodomains contributing to mechanisms by which cells can target growth factor activity (Steffensen et al., 2011). Studies using this approach have shown that cleavage of the ectodomain of acidic fibroblast growth factor receptor 1 (FGFR-1) by MMP-2 releases the entire extracellular domain of the receptor which then enables the binding and sequestration of fibroblast growth factor (FGF) in the tissue, suggesting that soluble forms of FGF-1 produced upon MMP-2 proteolysis contribute to angiogenic and mitogenic activities of FGF (Levi et al., 1996).

In summary, the complex structure and function of the ECM as well as the interaction of its molecular constituents with cells regulate numerous cellular fate processes such as adhesion, proliferation, migration, and differentiation. This dynamic cellular microenvironment is continuously being remodeled especially during the natural processes of development, differentiation, and wound repair. Understanding the complex dynamic relationship between cells and the ECM is critical to the successful design of synthetic biomaterial scaffolds that promote tissue regeneration.

\section{Synthetic PEG-based ECMs for the support of 3D cell and tissue growth}

Naturally derived as well as synthetic hydrogels have been extensively investigated in tissue engineering. Natural hydrogels are primarily obtained from ECM proteins (e.g., collagen, fibrin) and polysaccharides (e.g., alginate, chitosan, hyaluronic acid, dextran) (Zhu, 2010). Collagen and fibrin are clinically well-established FDA-approved materials for the healing of burns and chronic wounds, and used as tissue sealants, respectively (Lutolf \& Hubbell, 2005). In general, hydrogels derived from natural sources are advantageous over synthetic hydrogel formulations since they possess the structural complexity and functional capacity of a particular tissue of interest. Despite these advantages, their use is often restricted due to complexities associated with purification, immunogenicity, and pathogen transmission (Patterson et al., 2010; Zhu, 2010). In addition, their mechanical properties cannot be readily manipulated, especially independent of alterations in their biochemical composition. These associated disadvantages of naturally-derived ECM components have resulted in a shift over the last several decades towards the use of synthetic biomaterials that 
offer greater and systematic control of material properties for the design of scaffolds of multiple tissue types.

Among the classes of synthetic biomaterials, PEG hydrogels have been extensively investigated as scaffolds in tissue engineering. Crosslinked PEG hydrogel networks swell extensively in aqueous environments providing a 3D highly swollen network with viscoelastic properties similar to soft tissues enabling diffusive transport and interstitial flow characteristics (Lutolf \& Hubbell, 2005). The physical and mechanical properties of these synthetic biomaterials can be readily manipulated via alterations in selected polymerization conditions that directly influence hydrogel crosslink density, swelling, and the elastic modulus resulting in scaffolds with rigidities ranging from those found in soft tissues such as the liver and skin up to rigidity values of articular cartilage and bone (Nemir \& West, 2010). PEG scaffolds are also biocompatible and intrinsically resistant to non-specific cell adhesion and protein adsorption. This inherently inert feature offers the advantage for selective incorporation of a variety of identified biofunctional oligopeptide sequences and proteins of the native ECM whose concentration and spatial distribution can be easily modulated within the crosslinked network to provide fundamental insight of signaling events involved in specific cell-matrix interactions. Finally, using a variety of different chemistries, PEG-based hydrogels can be polymerized under mild physiological conditions in the presence of cells and in situ with negligible loss in cell viability and function (Nuttelman et al., 2006; Underhill et al., 2007).

\subsection{Mechanisms of biofunctional PEG hydrogel formation}

While various forms of gelation exist (i.e., physical, ionic, and covalent interactions), covalent crosslinking leads to more stable PEG hydrogel networks with tunable physical and mechanical properties (Lin \& Anseth, 2009). Here we focus our attention on the various mechanisms of covalent crosslinking used for the fabrication of PEG hydrogel ECM mimics as well as the chemistries employed to engineer biofunctional monomers containing key signaling molecules of the native ECM that can be copolymerized and subsequently immobilized within PEG hydrogel networks.

\subsubsection{Mechanisms of covalently crosslinked hydrogel formation}

The synthesis of covalently crosslinked PEG hydrogels falls into one of three major categories: chain growth or free-radical photopolymerization, step-growth polymerization, and mixed-mode polymerization (a combination of chain and step growth reactions). Chain growth reactions such as those occurring in free-radical photopolymerization are initiated upon the photocleavage of initiator molecules in the presence of UV or visible light to yield primary radical species. These free-radicals propagate through unsaturated vinyl or acrylate bonds on PEG macromers. Covalent crosslinking and subsequent gelation is induced in the presence of PEG macromers containing multiple acrylate groups as in the case of the crosslinking agent PEG diacrylate (PEGDA). In these systems, complete gelation is achieved at relatively short times (on the order of seconds up to a few minutes). Photopolymerization of PEGDA hydrogels has been extensively investigated in tissue engineering and regenerative medicine applications, especially since it has been found as a suitable approach for the in situ encapsulation of proteins and cells. 
Step-growth polymerization and gelation occurs when at least two multifunctional macromers that contain complementary reactive chemical groups are reacted under stoichiometric balanced or imbalanced ratios. This polymerization can proceed under physiologic conditions without the use of free-radical initiators and permits more precise control over the network crosslink density (Lin \& Anseth, 2009). Hubbell and colleagues have developed a step-growth approach to form crosslinked PEG hydrogel networks that proceed via a Michael-type addition reaction using acrylated star PEG polymers and dithiol monomers (Elbert et al., 2001; Rizzi et al., 2006; Rizzi \& Hubbell, 2005). This polymerization mechanism was later extended to fabricate PEG hydrogel ECM mimics using multiarm PEG vinyl sulfone macromers (n-PEG-VS) to allow a Michael-type addition reaction between acrylated PEG and thiol groups presented on free cysteine amino acids on peptides and proteins (Raeber et al., 2005). Michael-type addition reactions are not limited to acrylates as they can also occur between maleimide and thiol groups (Patterson et al., 2010). As compared to free-radical polymerization times, Michael addition reactions take longer to complete (on the order of hours). Another type of stepgrowth gelation mechanism recently employed for the fabrication of PEG hydrogels is Click chemistry. This approach has elicited tremendous interest in tissue engineering due the well-defined network structures created, improved mechanical properties, its resulting versatility with respect to bioconjugation, and enhanced swelling capacities over other gelation approaches (DeForest et al., 2009; Malkoch et al., 2006; Polizzotti et al., 2008). In this method of gelation, macromers bearing azide and alkyne functional groups are "clicked" together in the presence of copper catalysts to form stable covalent linkages after which biofunctional molecules can be conjugated (Lin \& Anseth, 2009). One major drawback with conventional Click PEG hydrogels is that the reactions are catalyzed by copper which is cytotoxic to cells and not suitable for their encapsulation during the gelation process. Recently, Bertozzi and coworkers have developed a copper-free-click chemistry approach which offers great potential for a variety of tissue engineering applications (Laughlin et al., 2008).

PEG hydrogels crosslinked using a mixed-mode approach have been developed by Anseth and colleagues using thiol acrylate photopolymerization (Salinas \& Anseth, 2008). In this approach the polymerization is initiated photochemically and proceeds via step growth reactions between thiol and acrylate groups followed by acrylate homopolymerization which proceeds by chain polymerization. The propagation of polymer chains in this mixed mode process of hydrogel formation involves three reactions: the first step involves the step-wise reaction of a thiyl radical with a vinyl group, the second step involves the chain transfer of a radical from a carbon intermediate to a thiol, and the third reaction involves the homopolymerization of the acrylate radical via chain growth propagation. Thiol acrylate polymerization involves the chain transfer of growing polymer chains to thiol monomers which do not occur during the stepwise mechanism of Michael-type addition reactions. Unlike chain growth polymerizations, the use of photoinitiators is not necessarily required in mixed mode photopolymerization. Finally, the competition between the acrylate homopolymerization with the step growth thiol acrylate reaction results in different kinetics with more versatile network structures than those formed with pure step growth or free-radical polymerization gelation chemistries (Lin \& Anseth, 2009). 


\subsubsection{Engineering ECM cues into PEG hydrogel scaffolds}

Throughout the last several decades, extensive research has been conducted to systematically incorporate cell signaling molecules of the native ECM within PEG scaffolds which have provided fundamental insight on specific cell-substrate interactions. As previously mentioned, in the absence of cell signaling molecules, PEG hydrogels are inert biocompatible matrices, which do not elicit cell adhesion or protein adsorption. This discovery along with the identification of small peptide sequences of ECM adhesion proteins (Ruoslahti, 1996) has led to their widespread use as matrices upon which to introduce key biofunctional signals of the native ECM in either soluble or covalently immobilized form for modulating specific cellular responses in vitro and in vivo.

The minimum requirement for anchorage-dependent cells to survive on and within PEG scaffolds is to render them cell-adhesive. Thus, numerous researchers have covalently incorporated cell adhesion sequences, the most common being the RGD sequence, within PEG hydrogels using a variety of conjugation approaches, as described below. However, the incorporation of cell adhesion sequences in PEG hydrogels does not enable threedimensional (3D) prolonged cell survival when cells are encapsulated within these scaffolds due to the mesh size of the crosslinked PEG network being less than the average diameter of a cell, which poses a physical barrier to the critical process of cell migration (Raeber et al., 2005). Therefore, studies investigating cell behavior on PEG hydrogels modified with cell adhesion ligands have been primarily limited to two dimensions (2D) (at the cell-substrate interface), which is not representative of the actual processes of cell behavior and tissue remodeling in 3D. Nonetheless, these studies have provided significant insight on the effects of scaffold properties on 2D cell behavior which have led to the future design of scaffolds in tissue engineering (DeLong et al., 2005 a, 2005 b; Guarnieri et al., 2010; Hern \& Hubbell, 1998).

The applicability of PEG hydrogels in promoting 3D cell behavior and tissue regeneration is to render the scaffolds degradable. This requires that material degradation rate be matched with the rate of tissue regeneration (Papavasiliou et al., 2010). Although various approaches have been used to fabricate degradable PEG hydrogels with pre-engineered degradation rates, as in the case where hydrolytically degradable segments of poly(lactic acid) PLA, poly(glycolic acid) PGA, or poly(lactic-co-glycolic acid) PLGA are copolymerized into the crosslinked network, the ability to modify hydrogel degradation rates post-gelation using this approach is limited (Lin \& Anseth, 2009). In addition, hydrolytic degradation is not representative of the cellularly mediated and dynamic process of proteolysis that takes place within the native ECM. Therefore, numerous studies have covalently incorporated peptide sequences susceptible to cleavage by cell-secreted proteases (i.e., plasmin-sensitive or MMPsensitive sequences) into PEG hydrogels thus manipulating gel degradation dynamically in response to cellularly mediated events (Gobin \& West, 2002; Lee et al., 2007; Lutolf et al., 2003; Moon et al., 2007; Moon et al., 2010; Patterson \& Hubbell 2010; Phelps et al., 2009; Seliktar et al., 2004; West \& Hubbell, 1999; Zisch et al., 2003).

In the native ECM, growth factors are stored and released from ECM proteoglycans upon cellular demand, or secreted by cells, thus playing critical roles in controlling cellular function and tissue regeneration (Chen \& Mooney, 2003). While one therapeutic approach would be the bolus delivery of growth factors to the damaged and or diseased tissue, 
growth factors have short half-lives in the circulation and their distribution and rapid degradation may result in undesirable systemic effects and toxicity (Patterson et al., 2010). An alternative approach is to encapsulate growth factors in scaffolds such as those of PEG; however, this has been shown to result in a rapid burst release during the initial hydrogel swelling phase (Zhu, 2010). Alternatively, growth factors can be covalently incorporated into PEG hydrogels in order to prolong their biological activity and to mimic growth factor binding to ECM proteins.

In an effort to covalently immobilize peptides and/or proteins into PEG hydrogels, the pioneering work of Hern et al. showed that the maintenance of biological activity of synthetic peptide sequences upon immobilization requires that the peptide be flexible and experience minimal steric hindrance (Hern \& Hubbell, 1998). This study also showed that the tethering of RGD was required for cells to adhere onto crosslinked PEGDA hydrogel surfaces that contained RGD groups modified with a PEG spacer arm (MW=3400) whereas hydrogel surfaces immobilized with RGD in the absence of a spacer exhibited limited cell adhesion. Other studies used different molecular weights of PEG analogues to investigate the effect of linker length on osteoblast adhesion and determined that the effective distance between the peptide and the substrate was $3.5 \mathrm{~nm}$ corresponding to an approximate length of a PEG of molecular weight of 3500 Da (Kantlehner et al., 1999). Later studies combined both tethered cell adhesion ligands and growth factors that included a PEG pacer into PEG hydrogels in order to enhance cell proliferation and migration on hydrogel surfaces (DeLong et al., 2005). Early studies by West and Hubbell synthesized PEG hydrogels containing collagenase- and plasmin-sensitive domains between crosslinks that would degrade upon proteolysis to mimic the natural process of ECM degradation (West \& Hubbell, 1999). These pioneering studies led to the synthesis of PEG hydrogel networks that incorporated a variety of different pendant cell adhesion sites and growth factors, and crosslinked enzyme-sensitive domains for studying a variety of cell-substrate interactions. The resulting polymer structure of a covalently crosslinked PEG hydrogel network with incorporated biofunctionality for promoting 3D cell proliferation, migration, and tissue regeneration is shown in Figure 2.

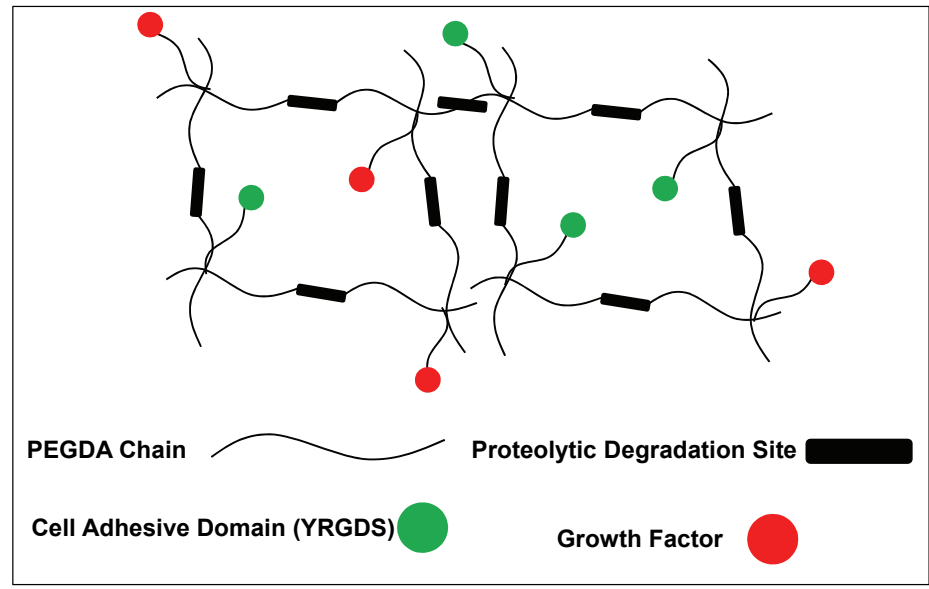

Fig. 2. Schematic of ECM signals immobilized in crosslinked PEG hydrogel scaffolds. 
Various conjugation approaches have been used to covalently immobilize cell adhesion ligands, proteolytically-sensitive domains, and growth factors within PEG hydrogels crosslinked via the gelation mechanisms described above. In PEGDA hydrogels formed by free-radical photopolymerization, biofunctionality is incorporated into the hydrogel network primarily through the copolymerization of peptide and/or protein monoacrylate and diacrylate macromers. These biofunctional macromers may be synthesized by reacting acryl-PEG-NHS (NHS = N-hydroxyl succinimide) with peptide resulting in the coupling of the $N$-terminal $\alpha$-amine of the peptide to the acrylate PEG macromer. In general the synthesis of biofunctional PEG monoacrylate macromers is achieved by reacting the AcrylPEG-NHS with peptide in a 1:1 mole ratio so that the resulting macromers are immobilized in a pendant fashion within the hydrogel network upon gelation as in the case where tethered cell adhesion and growth factors are desired. The synthesis of biofunctional PEG diacrylate macromers results via the reaction of the Acryl-PEG-NHS derivative with peptide in a 2:1 mole ratio to produce Acryl-PEG-peptide-PEG-Acryl. This biofunctional PEGDA peptide conjugate therefore becomes crosslinked within the hydrogel network upon polymerization as in the case of crosslinked enzymatically degradable peptide sequences. The physical (swelling ratio), mechanical (elastic modulus), and degradative properties of biofunctional PEG hydrogels can be manipulated by adjusting the polymerization conditions such as the molecular weight of the PEG spacer, or the PEGDA prepolymer weight percentage. Recently, an alternative approach has been used to synthesize crosslinkable PEGDA peptide conjugates by Michael-type addition reaction (Miller et al., 2010). This conjugation involves the step-wise reaction of bis-cysteine peptides with PEGDA to make high molecular weight PEGDA macromer peptide conjugates by controlling the stoichiometric ratio of the functional groups (Tibbitt \& Anseth, 2009). These biofunctional PEG diacrylates could then be crosslinked via free-radical photopolymerization to form hydrogels susceptible to cleavage by cell-mediated proteolysis. The techniques mentioned above are not ideal for synthesizing PEG-peptide macromer conjugates since they often result in the formation of multiple types of macromer species of various molecular weights and reactive functionality that alter the kinetics of crosslinking and affect the hydrogel mechanical and physical properties. In order to circumvent these issues more extensive purification methods may be required to separate these undesired products postconjugation and prior to polymerization.

Step-growth gelation mechanisms such as Michael-type addition allow for facile incorporation of cysteine-containing peptides with various functionalized types of PEG or multiarm PEG macromers (i.e., acrylate, maleimide, and vinyl-sulfone) in a one-step process. For example, Hubbell and colleagues have reacted multi-arm PEG vinyl sulfone with thiol groups of cysteine RGD containing peptides as well as dithiol bearing enzymatically degradable peptide sequences to form crosslinked cell adhesive and proteolytically degradable PEG hydrogels (Lutolf \& Hubbell, 2005; Patterson \& Hubbell 2010; Raeber et al., 2005; Zisch et al., 2003).

In the mixed mode gelation processes of thiol-acrylate photopolymerization, Anseth and coworkers have synthesized biofunctional PEG hydrogels via the use of thiol-bearing peptides (Lin \& Anseth, 2009; Salinas \& Anseth, 2008). Since thiol bearing compounds act as chain transfer agents during free-radical polymerization, the use of cysteine-containing peptides enables the peptides to act as chain transfer agents resulting in their incorporation 
within the hydrogel network. As compared to the bioconjugation approaches of pure freeradical photopolymerization described above, this mixed-mode approach does not rely on the additional step of synthesizing the acrylate and diacrylate PEG-peptide conjugates. Rather, the peptides are directly tethered in a single step fashion within the hydrogel network during photopolymerization as in the case of Michael-type addition.

\subsection{Mechanical properties and incorporated biofunctionality of proteolytically- sensitive PEG hydrogels influence 3D cell behavior and tissue regeneration}

PEG hydrogels immobilized with cell adhesive peptides, growth factors, and enzymesensitive peptides have been widely investigated as ECM mimics for numerous tissue engineering applications. A number of enzyme-sensitive peptides have been incorporated into PEG hydrogels such as collagen-derived GPQG $\downarrow$ IAGQ or MMP-sensitive (Lutolf et al., 2003),

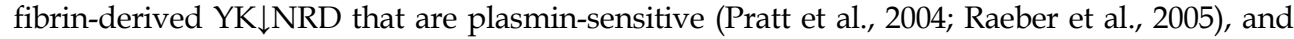
elastase-sensitive peptides such as AAPV $\downarrow$ RGGG (Aimetti et al., 2009), where $\downarrow$ denotes the peptide cleavage site. Here, we discuss the effects of hydrogel mechanical properties, proteolytically mediated degradation rate, and growth factor presentation on 3D cell behavior and tissue regeneration within PEG hydrogels immobilized with MMP-sensitive domains. We limit our attention to MMP-sensitive PEG hydrogels due to the importance of MMP enzymes in critical processes such as wound healing and vascularization.

Numerous studies have shown that the 3D presentation of specific soluble and immobilized biofunctional cues as well as the mechanical properties (Bott et al. 2010) of biofunctional PEG scaffolds have a profound impact on in vitro (Leslie-Barbick et al., 2009; Saik et al., 2011; Seliktar et al., 2004; Zisch et al., 2003) and in vivo (Moon et al., 2010; Phelps et al., 2009 ; Zisch et al., 2003) cell behavior. Our recent data (unpublished results) illustrate that the degradation rate of MMP-sensitive PEGDA hydrogels formed by free-radical photopolymerization in the presence of visible light $(\lambda=514 \mathrm{~nm})$ can be controlled through variations in the weight percent of the MMP-sensitive PEG diacrylate macromer when incubated in collagenase enzyme solution. Increases in the weight percentage of the MMPsensitive diacrylate increases the presentation of MMP-sensitive domains in the network, but also results in a higher crosslink density and in decreased diffusion of enzyme in the gel, thus increasing hydrogel degradation times (Fig. 3).

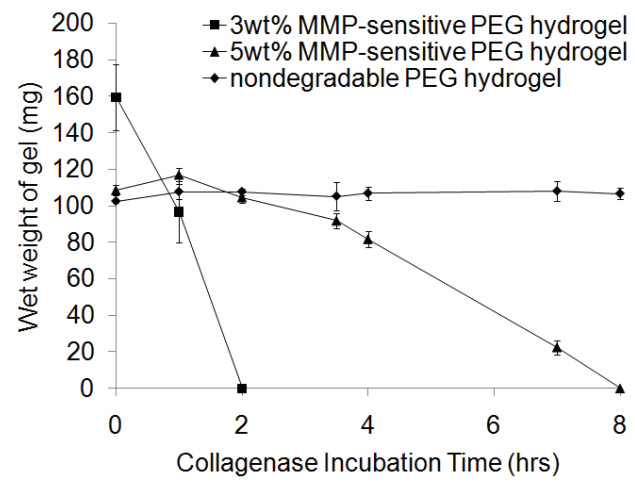

Fig. 3. Degradation profiles of photopolymerized MMP-sensitive PEGDA hydrogels. 
For hydrogels that display the fastest degradation, the encapsulation of fibroblasts within PEGDA scaffolds immobilized with MMP-sensitive crosslinks and tethered RGD results in robust invasion of the PEG matrix after 2 weeks in culture (Fig. 4). Other studies using

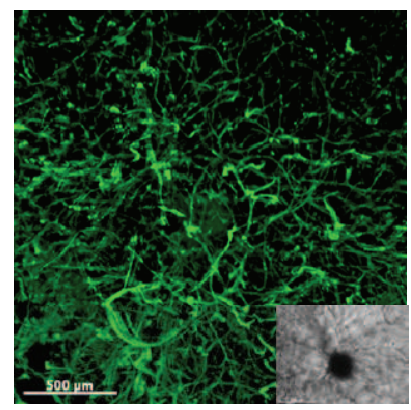

Fig. 4. Fibroblast invasion within an MMP-sensitive PEGDA hydrogel matrix 2 weeks postencapsulation. Scale bar $=500 \mu \mathrm{m}$.

Michael-type addition for the fabrication of PEG hydrogels immobilized with collagenbased MMP-sensitive sequences and the cell adhesion ligand RGD showed that the proliferation of fibroblasts can be manipulated via changes in the hydrogel modulus (ranging from 250 to $1100 \mathrm{~Pa}$ ) regardless of the sensitivity of the PEG matrix to proteolysis and the presence of cell adhesion motifs (Bott et al., 2010). Studies using UV-based freeradical photopolymerization for the formation of MMP-sensitive, VEGF-bearing PEGDA hydrogels as potential matrices for the induction of vascularization indicated that endothelial cell tubule formation and maintenance was directly affected by gel mechanical properties. Hydrogels formed with an intermediate polymer weight percentage of $10 \%$ resulted in the formation of tubules, while less crosslinked PEGDA matrices (7.5\% PEG content) caused complete tubule regression, and matrices (15\% PEG content) reduced tubule formation in vitro (Moon et al., 2010). PEG hydrogels of this intermediate stiffness were implanted into mouse cornea using a micropocket angiogenesis assay and resulted in neovascularization in the presence of vascular endothelial growth factor (VEGF) (Rogers et al., 2007). In this study, the stiffness of the hydrogel and sensitivity to proteolysis were simultaneously altered through variations in the weight percentage of the MMP-sensitiveconjugated PEGDA macromer with compressive moduli ranging from 30 to $110 \mathrm{kPa}$. This study and others have demonstrated that hydrogel degradation and the formation of functional blood vessels within biofunctional PEG hydrogels can be manipulated by changing the biophysical properties of the hydrogel.

While the physical and mechanical properties of PEG hydrogels play a critical role in dictating 3D cell behavior, extensive research has been conducted on investigating the effects of growth factor identity and delivery on cell behavior and tissue regeneration within PEG scaffolds. The successful application of PEG scaffolds is highly dependent on their ability to promote stable neovascularization (new blood vessel formation) to meet the in vivo oxygen and nutrient demands associated with tissues (Papavasiliou et al., 2010). Growth factors, such as VEGF and the fibroblast growth factor (FGF) families, in addition to MMPs, play critical roles during vascularization. Therefore, these biofunctional signals have been incorporated into PEG scaffolds to study their effects on vascularization in vitro and in vivo. 
Growth factors have been incorporated into PEG scaffolds in either soluble or immobilized form or in combinations. For example, MMP-sensitive PEG hydrogels were investigated as a bioactive co-encapsulation system for vascular cells and thymosin $\beta 4$, a small bioactive molecule. This system was able to induce adhesion, survival, migration and organization of human umbilical vein endothelial cells as well as the controlled release of thymosin $\beta 4$ triggered by MMP-2 and MMP-9 enzymes (Kraehenbuehl et al., 2009). In a similar study, collagenase-degradable PEG hydrogels modified with covalently immobilized VEGF and RGDS showed a fourteen-fold increase in endothelial cell motility and a three-fold increase in cell-cell connections in the presence of VEGF (Leslie-Barbick et al., 2009). While the abovementioned studies have focused on the presentation of a single growth factor to cells encapsulated in PEG hydrogels, multiple growth factors are required to drive the process of tissue regeneration to completion in vivo (Chen \& Mooney, 2003). Therefore, PEGDA hydrogel studies have recently investigated the effects of immobilized combinations of multiple growth factors on the ability of cells to induce key responses involved in angiogenesis (Saik et al., 2011). The immobilization of two key angiogenic proteins, plateletderived growth factor-BB (PDGF-BB) and fibroblast growth factor-2 (FGF-2), resulted in increased endothelial cell migration in vitro compared with the immobilization of each factor alone. Furthermore, the combination of soluble PDGF-BB and immobilized PDGF-BB induced a more robust vascular response compared with soluble PDGF-BB alone when the hydrogels were implanted in vivo in a mouse cornea micropocket angiogenesis assay.

In the native ECM, growth factors are released by cell-associated enzymatic activity that degrades the matrix, or that cleaves the matrix binding domain between the growth factor and the ECM component proteins (Zisch et al., 2003). To mimic a delivery system whereby the rate of growth factor release could be controlled by matrix degradation and by cleavage of the matrix-growth factor binding site, PEG hydrogels were engineered with MMPsensitive domains as well as with immobilized VEGF that was designed to contain an enzymatically degradable plasmin linker. This plasmin linker would allow for VEGF release in response to plasmin secretion as principal forms of VEGF are known to contain a binding site for heparin sulfate proteoglycans which maintain VEGF in its immobilized state until released from local cellular enzymes (Zisch et al., 2003). These matrix associations stabilize the growth factor in its active conformation protecting it from proteolytic inactivation. When these hydrogels were implanted subcutaneously in rats, they were completely remodeled into native, vascularized tissue.

Most of the studies focusing on proteolytically degradable PEG hydrogels for tissue engineering applications have primarily utilized the MMP-sensitive substrate site found within the $\alpha$-chain of type I collagen (GPQG $\downarrow$ IAGQ or GPQG $\downarrow I W G Q)$. (Seliktar et al., 2004) However, these substrates do not degrade particularly fast which may limit cellular infiltration within the scaffold and these peptides can be also be cleaved by a variety of MMPs. Thus, recent strategies have focused on enhancing proteolytic degradation of PEG hydrogels by targeting peptide substrates with increased catalytic activity (Patterson \& Hubbell, 2010, 2011) or by increasing the spatial presentation of these signaling molecules within the hydrogel network (Miller et al., 2010). To increase the spatial presentation, studies have used step-growth polymerization of PEG diacrylate and bis-cysteine MMPsensitive peptides and via Michael-type addition formed biodegradable ultra-high

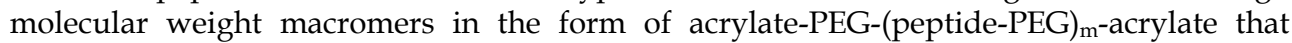
when photopolymerized into hydrogels resulted in increased swelling and degradability 
compared to previously published systems. This system induced angiogenesis in an ex vivo aortic arch explant assay and demonstrated that capillary sprouting can be tuned by engineering the susceptibility of the hydrogels to MMP enzymes and the adhesive ligands (Miller et al., 2010). Additionally, using combinatorial methods of peptide libraries, MMP substrate sequences that show increased enzymatic degradation and specificity have been identified (Patterson \& Hubbell, 2010, 2011; Turk et al., 2001). In an effort to enhance proteolytic degradation within these synthetic matrices, one study screened these substrates for enhanced degradability by MMP-1 and MMP-2 enzyme solutions in soluble form and as biodegradable crosslinkers within PEG hydrogels (Patterson \& Hubbell, 2010). The study showed that increases in catalytic activity resulted in faster degradation times of the hydrogels formed with the different peptides and that certain peptides were more specific to either MMP-1 or MMP-2, while others were susceptible to both enzymes. In another study, these peptides were screened for plasmin sensitivity in addition to MMP-1 and MMP-2 to enhance hydrogel degradation. Hydrogels that displayed increased sensitivity to these enzymes resulted in faster fibroblast proliferation and greater cell invasion from aortic ring segments (Patterson \& Hubbell, 2011). These hydrogel systems allow tuning to enzymes that are produced by specific cell types giving a broader in vivo application.

\section{Biofunctional and mechanical gradients in PEG hydrogels}

While the above studies focused on investigating the effects of biofunctional ECM signals and mechanical properties that can be homogeneously distributed within PEG hydrogels on cell behavior, the spatial presentation of these factors plays a critical role in directing cell behavior and tissue regeneration. For example, critical cellular fate processes involved in tissue regeneration, such as cell migration, are directed by the complex spatial and temporal presentation of physical and chemical signals found in the ECM (Barkefors et al., 2008; Lo et al., 2000; Papavasiliou et al., 2010; Smith et al., 2009). Cellular responses to gradients of different stimuli can be separated into durotaxis, the process in which cells respond to changes in matrix rigidity (Lo et al., 2000), haptotaxis, a response to matrix bound chemoattractants (Lo et al., 2000; Smith et al., 2004; Kim et al., 2009), and chemotaxis, a response to a soluble factor that diffuses freely from its source (Barkefors et al., 2008; Lo et al., 2000). In vivo, studies of the retina have shown that gradients of secreted VEGF help shape vascular patterns during angiogenic sprouting by guiding endothelial tip cell formation and subsequent migration (Gerhardt et al., 2003). Furthermore, computational models predict that VEGF gradients in hypoxic muscle tissue are capable of directing the formation of new vessels (Mac Gabhann et al., 2007). Other studies have demonstrated that gradients of platelet derived growth factor (PDGF) guide fibroblasts towards wounded regions (Schneider et al., 2010) and that leukocytes migrate to areas of inflammation, infection or injury in response to secreted chemokines and bacterial byproducts (Moissoglu \& Schwartz, 2006). To date, a limited number of studies have sought to recreate both haptotactic and durotactic gradients within synthetic PEG scaffolds. PEG hydrogels with gradients of biofunctional ECM signals and/or stiffness have been generated using gradient makers (DeLong et al., 2005a, 2005b; Nemir \& West, 2010), microfluidic techniques (Burdick et al., 2004; Guarnieri et al., 2008, 2010) and perfusion-based frontal polymerization (Turturro \& Papavasiliou, 2011). In other instances, soluble growth factors have been included in PEG hydrogels to stimulate a chemotactic response as they diffuse from the implanted hydrogels into surrounding tissue (Papavasiliou et al., 2010). 


\subsection{Gradient makers}

The use of gradient makers to generate spatial variation in either matrix stiffness or covalently immobilized biofunctional moieties is attractive since these devices are easy to use and commercially available. Gradient makers were originally developed for the separation of proteins via gel electrophoresis but have been miniaturized to form biofunctional hydrogel gradients. This approach of gradient generation uses two feed streams containing different prepolymer compositions that are combined in varying proportions as they pass through a control valve. The mixture is gently added to a mold such that layers of varying prepolymer composition are retained (Fig. 5). The prepolymer solution is then converted into a hydrogel by photopolymerization resulting in a uniform gradient that transitions from one of the prepolymer compositions to the other. In one study, a gradient maker was employed to generate a gradient of covalently immobilized basic fibroblast growth factor (bFGF) by adding bFGF to only one of the feed streams (DeLong et al., 2005). Using this technique, it was shown that smooth muscle cells migrated towards regions of increasing concentration of bFGF. In another study, PEGDA hydrogels with gradients in elastic modulus ranging from $100 \mathrm{kPa}$ to 5 $\mathrm{kPa}$ were formed with a gradient maker using feed streams containing PEGDA macromers of two molecular weights, $3.4 \mathrm{kDa}$ and $20 \mathrm{kDa}$, respectively (Nemir et al., 2010). This study showed that that macrophages seeded on PEG hydrogel surfaces primarily adhered to regions of greater stiffness.

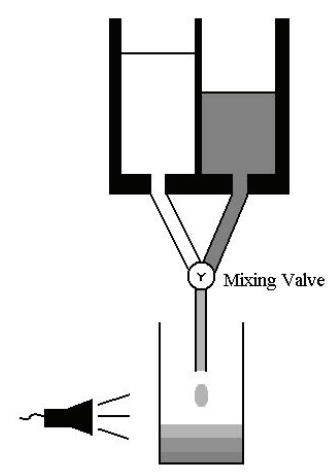

Fig. 5. Schematic illustration of gradient generation in photopolymerized PEG hydrogels using gradient makers.

\subsection{Microfluidics}

Similar to gradient makers, microfluidic techniques are based on the mixing of two prepolymer feed streams at varying ratios. The difference is that microfluidics uses a series of interconnected microchannels to intermix the feed streams and simultaneously create multiple layers each with a unique composition (Fig. 6). The resultant layers are then converted to a hydrogel by bulk photopolymerization. Using this approach, Burdick et al. combined two monomer solutions (with and without RGDS) to create gradients of RGDS (Burdick et al., 2004). They demonstrated that endothelial cell surface attachment increased with increasing immobilized RGDS content in PEGDA hydrogels formed by free-radical photopolymerization. Furthermore, when two different solutions of varying PEGDA 


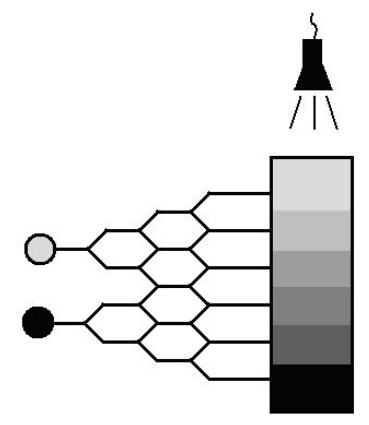

Fig. 6. Illustration of a microfluidic device for the generation of gradients in photopolymerized PEG hydrogel scaffolds.

molecular weight and concentration were injected into the microfluidic chamber, hydrogels with gradients of thickness were produced, suggestive of a gradient in crosslink density Other studies using microfluidic gradient makers have demonstrated directional fibroblast alignment (Guarnieri et al., 2008) and migration (Guarnieri et al., 2010) on PEGDA hydrogel surfaces with gradients of immobilized YRGDS. In the latter study, 2D cell migration was found to increase with increases in the slope of the gradient of immobilized RGD (Guarnieri et al., 2010).

\subsection{Perfusion based frontal polymerization}

In an effort to generate hydrogel scaffolds with controllable gradients of mechanical properties and incorporated biofunctionality, Turturro et al. have developed a novel freeradical photopolymerization technique, perfusion based frontal photopolymerization (PBFP) (Turturro \& Papavasiliou, 2011), which is a variant of a previously developed frontal polymerization process (Chechilo et al., 1972). Originally reported in the 1970's for the synthesis of poly(methyl methacrylate) under high pressure, frontal polymerization is based on the generation of a localized reaction zone that propagates through a monomeric mixture in a fashion similar to a reaction wave (Alzari et al., 2009; Chekanov \& Pojman, 2000). The reaction front is created by an external energy source, such as heat or light, and propagates through the system via consumption of the monomeric species. The PBFB technique is distinct from the previously published methods of frontal polymerization (Chechilo et al., 1972; Chekanov \& Pojman, 2000; Pojman et al., 1996) in that it is based on the perfusion of a photoinitiator resulting in localized initiation and subsequent formation of crosslinked PEGDA hydrogels with gradients of mechanical properties and of immobilized YRGDS. In PBFP, gradients are generated by the controlled delivery of eosin $Y$, a photoinitiator, to the base of the monomeric solution via a perfusion pump and a glass frit filter disk. Due to density differences between the PEGDA precursor solution and the eosin $Y$ photoinitiator, buoyant eosin $\mathrm{Y}$ rises to the surface and initiates propagation upon exposure to visible light $(\lambda=514 \mathrm{~nm})$. Additional photoinitiator is trapped by the polymer layer causing a descending polymer front that is characteristic of frontal polymerization (Fig. 7). Through controlled delivery of the photoinitiator and alteration of the polymerization conditions, this technique allows for manipulation of the magnitude of the gradients generated and is capable of producing multiple gradients in photopolymerized PEGDA scaffolds. As shown 


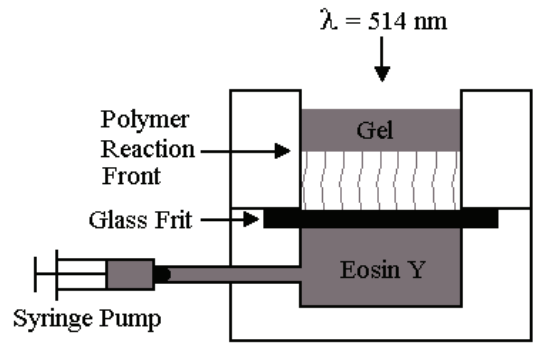

Fig. 7. Schematic of perfusion-based frontal polymerization.

in Figure 8, simultaneous gradients of elastic modulus and immobilized concentration of the YRGDS adhesion ligand are achieved using PBFP. In vitro cell studies in which aggregates of 3T3 fibroblasts are seeded on the surface of these hydrogels indicated that cells are capable of detecting and responding to the gradients generated via directional outgrowth. As shown in Figure 9, cells seeded on PBFP hydrogels spread roughly twice as far in the direction parallel to the gradient as in the perpendicular direction.

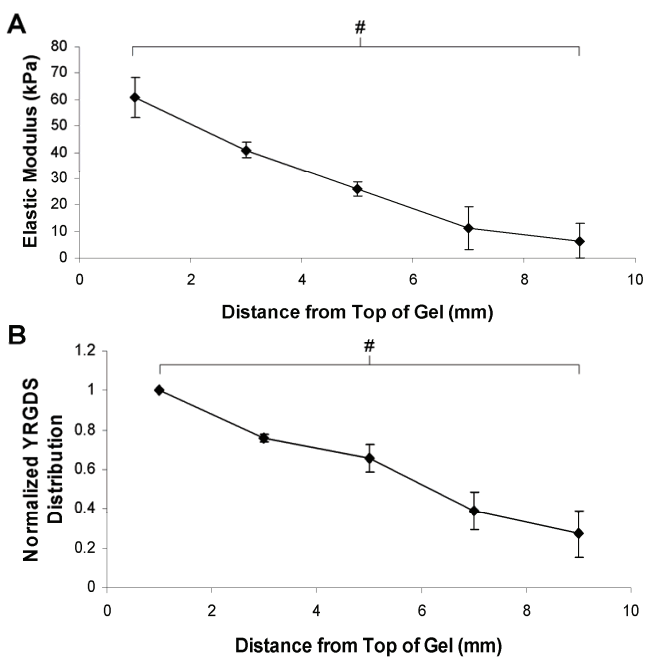

Fig. 8. Distribution of (A) elastic modulus and (B) immobilized YRGDS in PBFP PEGDA hydrogels $(n=3 ; \#=p<0.001)$.

\subsection{Microsphere packing}

In addition to the above mentioned techniques, gradients in PEG hydrogels have also been formed by microsphere packing (Roam et al., 2010). This technique relies upon the formation of PEG microspheres of varying density by thermally induced phase separation. The microspheres of varying density are combined in a serum rich solution and centrifuged to form a density gradient. The presence of the serum proteins allows the microspheres to 


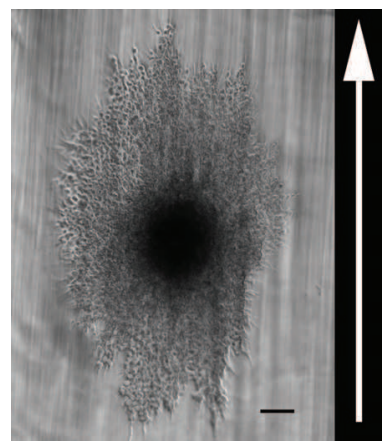

Fig. 9. Aggregate of $3 \mathrm{~T} 3$ fibroblasts 13 days post-seeding on a PFBP PEGDA hydrogel. The arrow indicates the direction of increasing elastic modulus and immobilized YRGDS concentration. Scale bar $=100 \mu \mathrm{m}$.

crosslink together and form solid hydrogels. Using this technique, PEG hydrogels containing gradients with either sharp interfaces or gradual transitions with up to 5 distinct layers were formed. This approach has been also used to fabricate macroporous PEG hydrogels with gradients in porosity and homogeneously distributed PEG microspheres loaded with RGD and sphingosine 1-phosphate that promoted endothelial cell infiltration through the scaffolds, but the effects of gradients on cell behavior have yet to be determined (Scott et al., 2010). This technique offers the potential for incorporating gradients of biofunctionality or even regions of different proteins/drugs that are capable of diffusing from a microsphere of a particular density and stimulating a desired cellular response.

The above mentioned techniques have proven successful in generating gradients of mechanical properties and biofunctional moieties that are capable of recreating the natural microenvironment of cells. However, the effects of PEG hydrogel gradients on cell behavior have been primarily limited to the cell-substrate interface and have not been quantified in 3D. Therefore the future of gradients will rest on the ability to combine gradient techniques with existing methods of incorporating degradable domains and in particular those susceptible to cell-mediated proteolysis within PEG hydrogels. Success in doing so will allow researchers to gain a better understanding of how the 3D spatial presentation of matrix signals influence cell behavior and tissue regeneration.

\section{Prediction of scaffold properties via computational hydrogel models}

In order to design a functional synthetic scaffold that supports $3 \mathrm{D}$ cell behavior, the mechanical properties, degradation kinetics, and spatial presentation of biochemical signals must be appropriately tuned prior to in vitro and/or in vivo cell studies (Tibbitt \& Anseth, 2009). Polymerization and hence gelation processes are kinetically controlled and consequently the resultant polymer properties are highly dependent on polymerization conditions. In systems where multiple biofunctional signals are to be incorporated into crosslinked PEG hydrogel networks, the number of reactions between functional monomeric species increases, and optimization of the physical, mechanical, and biofunctional spatial and temporal presentation of these matrix cues can become challenging for engineering specific tissues. The future design of PEG scaffolds would greatly benefit 
from computational models of biofunctional hydrogel formation that accurately predict the final concentrations of immobilized ECM peptide signals as well as the mechanical properties as a function of space and time due to alterations in polymerization conditions (e.g., initial concentrations of biofunctional monomers, polymerization time, polymer content). The predictive capability of these models would provide alternative approaches to detailed experimental sensitivity analysis for optimization of cell-substrate interactions.

A computational model of hydrogel formation of multiarm comb PEG crosslinked with transglutaminase enzyme has been developed on the basis of the Flory-Stockmyer theory (Sperinde \& Griffith, 2000). Gelation kinetics were predicted in terms of variations in the macromer structure and composition, stoichiometric ratios of reactants, and crosslinking enzyme concentration. Model predictions correlated well with gelation times with alterations induced via variations in enzyme concentration. These models, however, are based on statistical approaches that make it difficult to characterize the dynamics of the complex process of gel formation.

Other studies of computational modeling of free-radical photopolymerization of crosslinked PEGDA hydrogels formed via interfacial photopolymerization have predicted the formation of crosslink density gradients within planar hydrogel surfaces as a function of space and time (Kizilel et al., 2006). These models were based on previous models of gel formation occurring via free-radical crosslinking of vinyl and divinyl monomers developed by Gossage (Gossage, 1997). The models utilized the Numerical Fractionation (NF) technique which avoids the numerical divergence of the second and higher molecular weight moments at the gel point (Teymour \& Campbell, 1992). Utilization of this technique in polymerization models of gelation has provided extensive insight of the resulting polymer molecular weight distribution, the dynamics of gelation, and the evolution of the crosslink density of the gel (Gossage 1997; Kizilel et al., 2007; Papavasiliou et al., 2002; Papavasiliou \& Teymour, 2003; Teymour \& Campbell, 1992). Computational models of interfacial polymerization of PEG hydrogels using the NF technique were later extended to account for the formation of crosslinked PEG hydrogel films immobilized with glucagon-like peptide-1 for the prediction of film thickness and crosslink density in designing membranes for the encapsulation of islet cells. While these hydrogel models have provided insight into the effects of polymerization conditions on hydrogel crosslinking, they have yet to be validated in terms of incorporated biofunctionality, and hydrogel crosslink density.

The future use and implementation of computational models of hydrogel formation for the design and optimization of scaffolds in tissue engineering will require that they be validated in terms of their mechanical and physical properties as well as the immobilized concentrations of multiple ECM signals. A critical parameter in validating these models will be the experimental determination of unknown kinetic rate constants that play a critical role in dictating the kinetics of polymerization. For hydrogels formed via free-radical photopolymerization, the incorporation of biofunctional-PEG macromer conjugates into PEG scaffolds formed using either Acryl-PEG-NHS chemistry or Michael-type addition reaction between bis-cysteine and PEGDA requires further optimization. These biofunctional macromer conjugation schemes can often result in multimodal molecular weight distributions (Miller et al., 2010) which can directly affect hydrogel crosslinking, gel degradation rate, and the resulting biomaterial properties. Therefore, computational modeling at the macromer level would provide significant insight in defining the synthesis 
conditions required to eliminate the need of undesired species that may compromise biomaterial properties.

\section{Conclusion}

Significant progress has been made using PEG hydrogel scaffolds as ECM mimics for supporting and directing cell behavior and tissue regeneration. Ongoing challenges however remain in PEG-based biomaterial strategies for the engineering of new tissues. Although numerous efforts have focused on investigating the effects of biological signal identity, gel degradation rate, and mechanical properties on cell behavior, little work has been done to independently tune these properties in order to isolate and quantify the individual effects of these factors on cell behavior. Coupling computational models with experimental data to identify conditions that result in independent tuning of these properties would provide significant insight on how PEG scaffolds could be better designed to enhance tissue regeneration prior to complete material degradation. The ability to stimulate neovascularization within PEG hydrogels is also critical for the clinical success of designing tissues that require the formation of a stable and functional vasculature. Research in this regard has focused on vascularization of small polymer scaffolds which do not represent the clinical volumes needed for the generation of vessels in large tissues and therefore, strategies for the design of scaffolds that promote vascularization of larger tissues are needed to address this limitation. The modulation of the spatial arrangement of ECM signals and scaffold mechanical and physical properties in PEG hydrogels in directing cell behavior in response to gradients has been primarily limited to the cell-substrate interface. Future studies quantifying the effects of 3D gradients of multiple matrix cues and how this influences cellular fate processes will provide significant insight in effectively designing PEG-based scaffolds with gradients for processes that require more rapid and guided cell migration and tissue regeneration. Finally, alternative polymer strategies where the delivery of growth factors and other biofunctional signals can be dynamically regulated by the scaffolds themselves and in combination with cellularly-mediated events are critical to the success of PEG hydrogels in tissue engineering. These design criteria need to be considered for the continued enhancement of these scaffolds for regenerative medicine applications.

\section{Acknowledgement}

The authors would like to acknowledge funding from the Pritzker Institute of Biomedical Science and Engineering at the Illinois Institute of Technology and the National Institutes of Health (Grant No. R21HL094916).

\section{References}

Aimetti, A.A.; Tibbitt, M.W., et al. (2009). Human neutrophil elastase responsive delivery from poly(ethylene glycol) hydrogels. Biomacromolecules, Vol. 10, No.6, pp. 1484-1489

Alzari, V.; Monticelli, O., et al. (2009). Stimuli responsive hydrogels prepared by frontal polymerization. Biomacromolecules, Vol. 10, No.9, pp. 2672-2677

Barkefors I.; Le Jan S., et al. (2008). Endothelial cell migration in stable gradients of vascular endothelial growth factor $A$ and fibroblast growth factor 2 - Effects on chemotaxis and chemokinesis. Journal of Biological Chemistry, Vol. 283, No.20, pp. 13905-13912

Bott, K.; Upton, Z., et al. (2010). The Effect of Matrix Characteristics on Fibroblast Proliferation in 3D Gels. Biomaterials, Vol. 31, No.32, pp. 8454-8464 
Alberts,B.; Johnson A.; Lewis, J.; Raff, M.; Roberts, K.; Walter, P. (2008). Molecular Biology of the Cell (5 $5^{\text {th }}$ Edition), Galrland Science, ISBN, 978-0-8153-4105-5, New York, New York

Burdick J.A.; Khademhosseini A., et al. (2004). Fabrication of Gradient Hydrogels Using a Microfluidics/Photopolymerization Process. Langmuir, Vol. 20, No.13, pp. 5136-5156

Chechilo, N.M.; Khvilivitskii, R.J., et al. (1972). On the Phenomenon of Polymerization Reaction Spreading. Doklady Akademii Nauk SSSR, Vol. 204, pp. 1180-1181

Chekanov, Y.A.\&Pojman, J.A. (2000). Preparation of Functionally Gradient Materials via Frontal Polymerization. J. Appl. Poly. Sci., Vol. 78, No.13, pp. 2398-2404

Chen R.R. \& Mooney D.J. (2003). Polymeric Growth Factor Delivery Strategies for Tissue Engineering. Pharmaceutical Research, Vol. 20, No.8, pp. 1103-1112

Daley W.P.; Peters S.B., et al. (2008). Extracellular Matrix Dynamics in Development and Regenerative Medicine. Journal of Cell Science, Vol. 121, No.3, pp. 255-264

DeForest C.A.; Polizzotti B.D., et al. (2009). Sequential Click Reactions for Synthesizing and Patterning Three-Dimensional Cell Microenvironments. Nature Materials, Vol. 8, pp. 659-654

DeLong S.A.; Gobin A.S., et al. (2005). Covalent Immobilization of RGDS on Hydrogel Surfaces to Direct Cell Alignment and Migration. Journal of Controlled Release, Vol. 109, No.1-3, pp. 139-148

DeLong S.A., Moon J.J., et al. (2005). Covalently Immobilized Gradients of bFGF on Hydrogel Scaffolds for Directed Cell Migration. Biomaterials, Vol. 26, No.16, pp. 3327-3234

Elbert D.L.; Pratt A.B., et al. (2001). Protein Delivery from Materials formed by Self-Selective Conjugate Addition Reactions. Journal of Controlled Release, Vol. 76, No.1-2, pp. 11-25

Gerhardt H.; Golding M., et al. (2003). VEFG Guides Angiogenic Sprouting Utilizing Endothelial Tip Cell Filopodia. The Journal of Cell Biology, Vol. 161, No.6, pp. 1163-1177

Gobin A.S. \&West J.L. (2002). Cell Migration Through Defined, Synthetic Extracellular Matrix Analogs. The FASEB Journal, Vol. 16, No.3, pp. 751-753

Gossage J.L.(1997). Numerical Fractionation Modeling of Nonlinear Polymerization, $\mathrm{PhD}$ Thesis, Department of Chemical Engineering, Insitute of Technology, Chicago, IL, USA

Guarnieri D.; Capua A.D., et al. (2010). Covalently Immobilized RGD Gradient on PEG Hydrogel Scaffold Influences Cell Migration Parameters Acta Biomaterialia, Vol. 6, No.7, pp. 2532-2539

Guarnieri, D.; Borzacchiello, A., et al. (2008). Engineering of Covalently Immobilized Gradients of RGD Peptides on Hydrogel Scaffolds: Effect on Cell Behavior. Macromololecular Symposia, Vol. 266, No., pp. 36-40

Hern, D.L. \& Hubbell, J.A. (1998). Incorporation of Adhesion Peptides into Nonadhesive Hydrogels Useful for Tissue Resurfacing. Journal of Biomedical Materials Research, Vol. 39, No.2, pp. 266-276

Kantlehner, M.; Finsinger, D., et al. (1999). Selective RGD-Mediated Adhesion of Osteoblasts at Surfaces of Implants. Angewandte Chemie, Vol. 38, No.4, pp. 560-562

Kizilel. S.; Papavasiliou, G., et al. (2007). Mathematical Model for Vinyl-divinyl Polymerization. Macromolecular Reaction Engineering, Vol. 1, No.6, pp. 587-603

Kizilel, S.; Pérez-Luna, V.H., et al. (2006). Mathematical Model for Surface-initiated Photopolymerization of Poly(ethylene glycol) Diacrylate Macromolecular Theory and Simulations, Vol. 15, No.9, pp. 686-700

Kraehenbuehl, T.P.; Ferreira, L.S., et al. (2009). Cell-responsive Hydrogel for Encapsulation of Vascular Cells. Biomaterials, Vol. 30, No.26, pp. 4318-4324

Laughlin, S.T.; Baskin, J.M., et al. (2008). In Vivo Imaging of Membrane-Associated Glycans in Developing Zebrafish. Science, Vol. 320, No.5876, pp. 664-667 
Lee, S.H.; Moon, J.J., et al. (2007). Poly(ethylene glycol) Hydrogels Conjugated with a Collagenase-sensitive Fluorogenic Substrate to Visualize Collagenase Activity During Three-dimensional Cell Migration. Biomaterials, Vol. 28, No.20, pp. 3163-3170

Leslie-Barbick, J.E.; Moon, J.J., et al. (2009). Covalently-immobilized Vascular Endothelial Growth Factor Promotes Endothelial Cell Tubulogenesis in Poly(ethylene glycol) Diacrylate Hydrogels. Journal of Biomaterial Science Polymer Edition, Vol. 20, No.12, pp. 1763-1779

Levi, E.; Miao, H.Q., et al. (1996). Matrix Metalloproteinase 2 Releases Active Soluble Ectodomain of Fibroblast Growth Factor Receptor 1. Proceedings of the National Academy of Sciences of the United States of America, Vol. 93, No.14, pp. 7069-7074

Lin, C.C. \& Anseth, K.S. (2009). PEG Hydrogels for the Controlled Release of Biomolecules in Regenerative Medicine. Pharmaceutical Research, Vol. 26, No.3, pp. 631-643

Lo, C.M.; Wang, H.B., et al. (2000). Cell Movement is Guided by the Rigidity of the Substrate. Biophysical Journal, Vol. 79, No.1, pp. 144-152

Lutolf, M.P. \& Hubbell, J.A. (2005). Synthetic Biomaterials as Instructive Extracellular Microenvironments for Morphogenesis in Tissue Engineering. Nature Biotechnology, Vol. 23, No.1, pp. 47-55

Lutolf, M.P.; Lauer-Fields, J.L., et al. (2003). Synthetic Matrix Metalloproteinase-sensitive Hydrogels for the Conduction of Tissue Regeneration: Engineering Cell-invasion Characteristics. Proceedings of the National Academy of Sciences of the United States of America, Vol. 100, No.9, pp. 5413-5418

Mac Gabhann, F.; Ji, J.W., et al. (2007). VEGF Gradients, Receptor Activation, and Sprout Guidance in Resting and Exercising Skeletal Muscle. Journal of Applied Physiology, Vol. 102, No.2, pp. 722-734

Malkoch, M.; Vestberg, R., et al. (2006). Synthesis of Well-defined Hydrogel Networks Using Click Chemistry. Chemical Communications, No.26, 2006, pp. 2774-2776

Miller, J.S.; Shen, C.J., et al. (2010). Bioactive Hydrogels Made from Step-growth Derived PEG-peptide Macromers. Biomaterials, Vol. 31, No.13, pp. 3736-3743

Moissoglu, K. \& Schwartz, M.A. (2006). Integrin Signalling in Directed Cell Migration. Biology of the Cell, Vol. 98, No.9, pp. 547-555

Moon, J.J.; Lee, S.H., et al. (2007). Synthetic Biomimetic Hydrogels Incorporated with Ephrin-A1 for Therapeutic Angiogenesis. Biomacromolecules, Vol. 8, No.1, pp. 42-49

Moon, J.J.; Saik, J.E., et al. (2010). Biomimetic Hydrogels with Pro-angiogenic Properties Biomaterials, Vol. 31, No.14, pp. 3840-3847

Nemir, S.; Hayenga, H.N., et al. (2010). PEGDA Hydrogels with Patterned Elasticity: Novel Tools for the Study of Cell Response to Substrate Rigidity. Biotechnolgy and Bioengineering, Vol. 105, No.3, pp. 636-644

Nemir, S. \& West, J.L. (2010). Synthetic Materials in the Study of Cell Response to Substrate Rigidity Annals of Biomedical Engineering, Vol. 38, No.1, pp. 2-20

Nuttelman, C.R.; Benoit, D.S.W., et al. (2006). The Effect of Ethylene Glycol Methacrylate Phosphate in PEG hydrogels on Mineralization and Viability of Encapsulated hMSCs. Biomaterials, Vol. 27, No.8, pp.

Papavasiliou, G.; Birol, I., et al. (2002). Calculation of Molecular Weight Distributions in Non-linear Free-radical Polymerization Using the Numerical Fractionation Technique. Macromolecular Theory and Simulations, Vol. 11, No.5, pp. 533-548

Papavasiliou, G.; Cheng, M., et al. (2010). Strategies for Vascularization of Polymer Scaffolds. Journal of Investigative Medicine, Vol. 58, No.7, pp. 838-844 
Papavasiliou, G. \&Teymour, F. (2003). Reconstruction of the Chain Length Distribution for Vinyl-divinyl Copolymerization using the Numerical Fractionation Technique. Macromolecular Theory and Simulations, Vol. 12, No.8, pp. 543-548

Papavasiliou, G.; Cheng, M.H., et al. (2010). Strategies for Vascularization of Polymer Scaffolds. Jourmal of Investigative Medicine, Vol. 58, No.7, pp. 838-844

Patino, M.G.; Neiders, M.E., et al. (2002). Collagen as an Implantable Material in Medicine and Dentistry. Oral Implantology, Vol. 28, pp. 220-225

Patterson, J. \& Hubbell, JA (2010). Enhanced Proteolytic Degradation of Molecularly Engineered PEG hydrogels in Response to MMP-1 and MMP-2. Biomaterials, Vol. 31, No.30, pp. 7836-7845

Patterson J.; Martino, M.M., et al. (2010). Biomimetic Materials in Tissue Engineering. Materials Today, Vol. 13, No.1-2, pp. 14-22

Patterson, J. \& Hubbell, J.A. (2011). SPARC-derived Protease Substrates to Enhance the Plasmin Sensitivity of Molecularly Engineered PEG hydrogels. Biomaterials, Vol. 32, No.5, (February, 2011), pp. 1301-1310

Phelps, E.A.; Landázuric N., et al. (2009). Bioartificial Matrices for Therapeutic Vascularization. Proceedings of The National Academy of Sciences of the United States of America, Vol. 107, pp. 3323-3328

Pojman, J.A.; Ilyashenko, V.M., et al. (1996). Free-radical Frontal Polymerization: SelfPropagating Thermal Reaction Waves. Journal of the Chemical Society, Faraday Transactions Vol. 92, No.16, pp. 2825-2837

Polizzotti, B.D.; Fairbanks, B.D., et al. (2008). Three-Dimensional Biochemical Patterning of Click-Based Composite Hydrogels via Thiolene Photopolymerization. Biomacromolecules, Vol. 2008, No.9, pp. 1084-1087

Pratt, A.B.; Weber, F.E., et al. (2004). Synthetic Extracellular Matrices for In Situ Tissue Engineering. Biotechnology and Bioengineering, Vol. 86, No.1, pp. 27-36

Raeber G.P.; Lutolf M.P., et al. (2005). Molecularly Engineered PEG hydrogels: A Novel Model System for Proteolytically Mediated Cell Migration. Biophysical Journal, Vol. 89, No.2, pp. $1374-1388$

Rizzi, S.C. \& Hubbell, J.A. (2005). Recombinant Protein-co-PEG Networks as Cell-Adhesive and Proteolytically Degradable Hydrogel Matrixes. Part I: Development and Physicochemical Characteristics. Biomacromolecules, Vol. 6, No.3, pp. 1226-1238

Rizzi, S.C.; Ehrbar, M., et al. (2006). Recombinant Protein-co-PEG Networks as CellAdhesive and Proteolytically Degradable Hydrogel Matrixes. Part II: Biofunctional Characteristics. Biomacromolecules, Vol. 7 No.11, pp. 3019-3029

Roam, J.L.; Xu, H. et al. (2010). The Formation of Protein Concentration Gradients Mediated by Density Differences of Poly(ethylene glycol) Microspheres. Biomaterials, Vol. 31, No.33, pp. 8642-8650

Rogers, M.S.; Birsner, A.E., et al. (2007). The Mouse Cornea Micropocket Angiogenesis Assay. Nature Protocols, Vol. 2, No.10, pp. 2545-2550

Ruoslahti, E. (1996). RGD and Other Regognition Sequences for Integrins. Annual Review of Cell and Developmental Biology, Vol. 12, pp. 697-715

Saik, J.E.; Gould, D.J., et al. (2011). Covalently Immobilized Platelet-derived Growth FactorBB Promotes Angiogenesis in Biomimetic Poly(ethylene glycol) Hydrogels. Acta Biomaterialia, Vol. 7, No.1, pp. 133-143

Salinas, C.N;. \& Anseth K.S. (2008). Mixed Mode Thiol-Acrylate Photopolymerizations for the Synthesis of PEG-Peptide Hydrogels. Macromolecules, Vol. 41, No.16, pp. 6019-6026

Saltzman, W.M. (2004). Tissue Engineering. Priniciples for the Design of Replacement Organs and Tissues. Oxford University Press, ISBN, 978-0-19-514130-6, New York, New York. 
Schneider, L.; Cammer, M., et al. (2010). Directional Cell Migration and Chemotaxis in Wound Healing Response to PDGF-AA are Coordinated by the Primary Cilium in Fibroblasts. Cellular Physiology and Biochemistry, Vol. 25, No.2-3, pp. 279-292

Scott ,E.A.; Nichols, M.D., et al. (2010). Modular Scaffolds Assembled Around Living Cells Using Poly(ethylene glycol) Microspheres with Macroporation via a Non-cytotoxic Porogen. Acta Biomaterialia, Vol. 6, No.1, pp. 29-38

Seliktar, D.; Zisch, A.H., et al. (2004). MMP-2 sensitive, VEGF-bearing Bioactive Hydrogels for Promotion of Vascular Healing. Journal of Biomedical Materials Research Part A, Vol. 68A, No.4, pp. 704-716

Singer, A.J. \& Clark, R.A. (1999). Cutaneous Wound Healing. New England Journal of Medicine, Vol. 341, No.10, pp. 738-746

Smith, J.T.; Kim, D.H., et al. (2009). Haptotactic Gradients for Directed Cell Migration: Stimulation and Inhibition Using Soluble Factors. Combinatorial Chemistry $\mathcal{E}$ High Throughput Screening, Vol. 12, No.6, pp. 598-603

Somerville, R.; Oblaner S., et al. (2003). Matrix Metalloproteinases: Old Dogs with New Tricks. Genome Biology, Vol. 4, No.216, pp. 1-11

Sperinde, J.J. \& Griffith, L.G. (2000). Control and Prediction of Gelation Kinetics in Enzymatically Cross-Linked Poly(ethylene glycol) Hydrogels. Macromolecules, Vol. 33, No.15, pp. 5476-5480

Steffensen, B.; Häkkinen, L., et al. (2011). Proteolytic Events of Wound-Healing Coordinated Interactions Among Matrix Metalloproteinases (MMPs), Integrins, and Extracellular Matrix Molecules. Critical Reviews in Oral Biology and Medicine, Vol. 12, No.5, pp. 373-398

Teymour, F. \& Campbell, J.D. (1992). Analysis of the Dynamics of Gelation in Polymerization Reactors Using the Numerical Fractionation Technique. Macromolecules, Vol. 27, No.9, pp. 2460-2469

Tibbitt, M.W. \& Anseth, K.S. (2009). Hydrogels as Extracellular Matrix Mimics for 3D cell Culture. Biotechnology and Bioengineering, Vol. 103, No.4, pp. 655-663

Turk, B.E.; Huang; L.L., et al. (2001). Determination of Protease Cleavage Site Motifs Using Mixture-Based Oriented Peptide Libraries. Nature Biotechnology, Vol. 19, No.7, pp. 661-667

Turturro, M.V. \& Papavasiliou, G. (2011) Generation of Mechanical and Biofunctional Gradients in PEG Diacrylate Hydrogels by Perfusion-Based Frontal Photopolymerization. Journal of Biomaterials Science Polymer Edition, http://www.ingentaconnect.com/content/vsp/bsp/pre-prints/jbs3298)

Underhill, G.H.; Chen, A.A., et al. (2007). Assessment of Hepatocellular Function within PEG hydrogels. Biomaterials 28, 256, 2007, Vol. 28, No.2, pp. 256-270

Vu, T.H. \& Werb, Z. (2000). Matrix metalloproteinases: Effectors of Development and Normal physiology. Genes and Development, Vol. 14, No.17, pp. 2123-2133

West, J.L. \& Hubbell, J.A. (1999). Polymeric Biomaterials with Degradation Sites for Proteases involved in Cell Migration. Macromolecules, Vol. 32, No.1, pp. 241-244

Zhu, J. (2010). Bioactive Modification of Poly(ethylene glycol) Hydrogels for Tissue Engineering. Biomaterials, Vol. 31, No.17, 2010), pp. 4369-4656

Zisch, A.H.; Lutolf, M.P., et al. (2003). Cell-demanded Release of VEGF from Synthetic, Biointeractive Cell-ingrowth Matrices for Vascularized Tissue Growth. The Journal of the Federation of Americal Societies of Experimental Biology, Vol. 17, No.13, 2003), pp. 2260-2262 


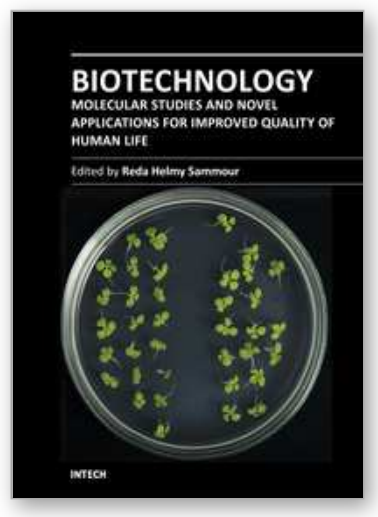

\author{
Biotechnology - Molecular Studies and Novel Applications for \\ Improved Quality of Human Life \\ Edited by Prof. Reda Sammour
}

ISBN 978-953-51-0151-2

Hard cover, 250 pages

Publisher InTech

Published online 14, March, 2012

Published in print edition March, 2012

This book deals with the importance of application of molecular biology as an approach of biotechnology for improvement of the quality of human life. One of the interesting topics in this field, is the identification of the organisms that produce bioactive secondary metabolites. It also discusses how to structure a plan for use and preservation of those species that represent a potential source for new drug development, especially those obtained from bacteria. The book also introduces some novel applications of biotechnology, such as therapeutic applications of electroporation, improving quality and microbial safety of fresh-cut vegetables, producing synthetic PEG hydro gels to be used as an extra cellular matrix mimics for tissue engineering applications, and other interesting applications.

\title{
How to reference
}

In order to correctly reference this scholarly work, feel free to copy and paste the following:

Georgia Papavasiliou, Sonja Sokic and Michael Turturro (2012). Synthetic PEG Hydrogels as Extracellular Matrix Mimics for Tissue Engineering Applications, Biotechnology - Molecular Studies and Novel Applications for Improved Quality of Human Life, Prof. Reda Sammour (Ed.), ISBN: 978-953-51-0151-2, InTech, Available from: http://www.intechopen.com/books/biotechnology-molecular-studies-and-novel-applications-for-improvedquality-of-human-life/synthetic-peg-hydrogels-as-extracellular-matrix-mimics-for-tissue-engineeringapplications

\section{INTECH}

open science | open minds

\section{InTech Europe}

University Campus STeP Ri

Slavka Krautzeka 83/A

51000 Rijeka, Croatia

Phone: +385 (51) 770447

Fax: +385 (51) 686166

www.intechopen.com

\section{InTech China}

Unit 405, Office Block, Hotel Equatorial Shanghai

No.65, Yan An Road (West), Shanghai, 200040, China

中国上海市延安西路65号上海国际贵都大饭店办公楼 405 单元

Phone: +86-21-62489820

Fax: $+86-21-62489821$ 
(C) 2012 The Author(s). Licensee IntechOpen. This is an open access article distributed under the terms of the Creative Commons Attribution 3.0 License, which permits unrestricted use, distribution, and reproduction in any medium, provided the original work is properly cited. 